\title{
THE DYNAMICAL MORDELL-LANG PROBLEM FOR NOETHERIAN SPACES
}

\author{
Jason P. Bell, Dragos Ghioca, Thomas J. Tucker
}

\begin{abstract}
Let $X$ be a Noetherian space, let $\Phi: X \longrightarrow X$ be a continuous function, let $Y \subseteq X$ be a closed set, and let $x \in X$. We show that the set $S:=\left\{n \in \mathbb{N}: \Phi^{n}(x) \in Y\right\}$ is a union of at most finitely many arithmetic progressions along with a set of Banach density zero. In particular, we obtain that given any quasi-projective variety $X$, any rational map $\Phi: X \longrightarrow X$, any subvariety $Y \subseteq X$, and any point $x \in X$ whose orbit under $\Phi$ is in the domain of definition for $\Phi$, the set $S$ is a finite union of arithmetic progressions together with a set of Banach density zero. This answers a question posed by Laurent Denis [7]. We prove a similar result for the backward orbit of a point and provide some quantitative bounds.
\end{abstract}

Keywords: dynamical Mordell-Lang conjecture, Noetherian space, Banach density.

\section{Introduction}

Motivated by the classical Mordell-Lang problem in arithmetic geometry, the following Conjecture was formulated in [11].

Conjecture 1.1. Let $X$ be any quasi-projective variety defined over $\mathbb{C}$, let $Y$ be any subvariety of $X$, let $\alpha \in X(\mathbb{C})$, and let $\Phi$ be an endomorphism of $X$. Then the set of $n \in \mathbb{N}$ such that $\Phi^{n}(\alpha) \in Y(\mathbb{C})$ is a finite union of arithmetic progressions.

We note that for an arithmetic progression we allow the possibility that its ratio equals 0 (in which case it is constant); so, in Conjecture 1.1 we allow the possibility of a finite intersection (which often is the case). Also we denote by $\mathcal{O}_{\Phi}(\alpha)$ the orbit of $\alpha$ under $\Phi$, i.e. the set consisting of all $\Phi^{n}(\alpha)$ for nonnegative integers $n$ (as always in algebraic dynamics, we denote by $\Phi^{n}$ the $n$-th iterate of $\Phi)$. In case of a rational self-map $\Phi$ we always work under the hypothesis that $\mathcal{O}_{\Phi}(\alpha)$ is entirely contained in the domain of definition for $\Phi$.

A reformulation of Conjecture 1.1 would be that if $Y$ is a subvariety of $X$ which contains infinitely many points of the form $\Phi^{n}(\alpha)$ (with $n \in \mathbb{N}$ ), then $Y$

\footnotetext{
The first and second authors were partially supported by NSERC. The third author was partially supported by NSF Grants DMS-0854839 and DMS-1200749.
}

2010 Mathematics Subject Classification: primary: 37P55; secondary: 11G99 
must contain a positive dimensional periodic subvariety under the action of $\Phi$, which has nontrivial intersection with $\mathcal{O}_{\Phi}(\alpha)$. This statement is in line with the classical Mordell-Lang problem, since in that case, if a subvariety $Y$ of a semiabelian variety contains infinitely many points from a finitely generated subgroup $\Gamma$, then $Y$ must contain a translate of an algebraic subgroup of positive dimension which has nontrivial intersection with $\Gamma$.

Conjecture 1.1 was proved in [2] for all étale endomorphisms $\Phi$ of any quasiprojective variety $X$ using a $p$-adic approach pioneered by the first author in [1]. Only special instances of Conjecture 1.1 when the map $\Phi$ is ramified are known (see $[4,5,13,23])$. In almost all known ramified cases, $\Phi$ is given by the coordinatewise action through one-variable rational maps on $\left(\mathbb{P}^{1}\right)^{m}$, i.e. $\Phi\left(x_{1}, \ldots, x_{m}\right)=$ $\left(\varphi_{1}\left(x_{1}\right), \ldots, \varphi_{m}\left(x_{m}\right)\right)$ for some rational maps $\varphi_{i}$. In particular, very little is known for arbitrary endomorphisms of quasi-projective varieties, besides the result of Fakhruddin [9], who proved that the Dynamical Mordell-Lang Conjecture holds for generic endomorphisms of $\mathbb{P}^{n}$. In this paper we obtain a very general result for Noetherian spaces in support of Conjecture 1.1. First we recall the definition of Banach density for subsets of $\mathbb{N}$, and then we define Noetherian topological spaces.

Definition 1.2. Let $S$ be a subset of the natural numbers. We define the Banach density of $S$ to be

$$
\delta(S):=\limsup _{|I| \rightarrow \infty} \frac{|S \cap I|}{|I|},
$$

where the limsup is taken over intervals $I$ in the natural numbers. We say that a subset $S$ of the natural numbers has Banach density zero if $\delta(S)=0$.

Definition 1.3. Let $X$ be a topological space. We say that $X$ is Noetherian if it satisfies the descending chain condition for its closed subsets, i.e., there exists no infinite descending chain of proper closed subsets.

Theorem 1.4. Let $X$ be a Noetherian topological space, and let $\Phi: X \longrightarrow X$ be a continuous function. Then for each $x \in X$ and for each closed subset $Y$ of $X$, the set $S:=\left\{n \in \mathbb{N}: \Phi^{n}(x) \in Y\right\}$ is a union of at most finitely many arithmetic progressions along with a set of Banach density zero.

In particular, Theorem 1.4 implies the following Corollary which provides evidence to an extension of the Dynamical Mordell-Lang Conjecture to the case of rational maps.

Corollary 1.5. Let $X$ be a quasi-projective variety defined over a field $K$, let $\Phi: X \longrightarrow X$ be a rational map defined over $K$, let $x \in X(K)$ such that $\mathcal{O}_{\Phi}(x)$ is contained in the domain of definition for $\Phi$, and let $Y$ be a $K$-subvariety of $X$. Then the set $S:=\left\{n: \Phi^{n}(x) \in Y(K)\right\}$ is a union of at most finitely many arithmetic progressions along with a set of Banach density zero.

Theorem 1.4 yields that the Dynamical Mordell-Lang principle almost holds for all continuous self-maps on Noetherian topological spaces. But obviously in 
this great generality it cannot always hold; we already know that if $X$ is a compact $p$-adic analytic manifold (endowed with the rigid analytic topology) and $\Phi$ is an analytic homomorphism, then the set $S$ might be infinite without containing an infinite arithmetic progression (see [3, Proposition 7.1]). Theorem 1.4 shows that once removing finitely many arithmetic progressions contained in $S$, we obtain a very sparse set. The key for our proof is the following Proposition which we state in the context of endomorphisms of quasi-projective varieties, but it is true in the more general context of continuous maps on Noetherian spaces (see Proposition 3.1).

Proposition 1.6. Let $X$ be a quasi-projective variety defined over the field $K$, let $x \in X(K)$, and let $\Phi: X \rightarrow X$ be an endomorphism defined over $K$. Assume $Y$ is a Zariski closed subset of $X$ with the property that the set $S:=\left\{n: \Phi^{n}(x) \in Y\right\}$ has positive Banach density. Then $S$ contains an infinite arithmetic progression.

Similar, but weaker, results were previously obtained in [7] and [3]. Denis [7] has treated the question of the distribution of the set $S$ when $Y$ does not contain a periodic subvariety. He showed, for any morphism of varieties over a field of characteristic 0, that $S$ cannot be very dense of order 2 (see [7, Définition 2]); this is a weaker conclusion than being of Banach density 0 (which is the result of our Proposition 1.6). We also note that Denis [7, Question 2] asked whether for endomorphisms $\Phi$ of quasi-projective varieties $X$, either the set $S$ (from Proposition 1.6 corresponding to a non-preperiodic point $x$ ) has Banach density 0, or the subvariety $V$ contains a positive dimensional periodic subvariety. Proposition 1.6 answers positively Denis' question since if $\delta(S)>0$ then $S$ contains an infinite arithmetic progression $\{a n+b\}_{n \in \mathbb{N}}$ and then $V$ would contain the Zariski closure of $\mathcal{O}_{\Phi^{a}}\left(\Phi^{b}(x)\right)$ whose positive dimensional components are periodic under $\Phi$. On the other hand, the result of [3] yields a stronger statement in terms of the sparseness of the set $S$ (assuming this set does not contain an infinite arithmetic progression); however the result of [3] applies only to endomorphisms of $\left(\mathbb{P}^{1}\right)^{n}$ of the form $\left(\varphi_{1}, \ldots, \varphi_{n}\right)$ where each $\varphi_{i}$ is a rational map defined over a field of characteristic 0 .

We note that due to the general setting of our Theorem 1.4 we are able to prove Corollary 1.5 for all rational maps on a quasi-projective variety, as opposed to regular maps only. We also note that our results apply in positive characteristic, in which case one knows that the Dynamical Mordell-Lang Conjecture fails (since also the classical Mordell-Lang principle fails in positive characteristic). For example, if $G$ is a semiabelian variety defined over $\mathbb{F}_{p}, C \subset G$ is a curve of genus greater than 1 defined over $\mathbb{F}_{p}$, and $\gamma \in C$ is a (generic) point not defined over $\overline{\mathbb{F}}_{p}$, then the intersection of $C$ with the cyclic subgroup of $G$ generated by $\gamma$ consists of all points of the form $p^{n} \gamma$ for nonnegative integers $n$.

As an easy consequence of our Corollary 1.5 we obtain the following results of Bézivin [6] and Methfessel [19].

Theorem 1.7. Let $r \geqslant 1$ be an integer, $K$ be a field, let $P_{0}, \ldots, P_{r} \in K[z]$ be polynomials such that $P_{r}(n) \neq 0$ for all $n \geqslant 0$, and assume the sequence $\left\{a_{n}\right\}_{n \geqslant 0} \subset$ 
$K$ satisfies the recurrence relation:

$$
P_{r}(n) \cdot a_{n+r}+P_{r-1}(n) \cdot a_{n+r-1}+\cdots+P_{0}(n) \cdot a_{n}=0,
$$

for all $n \geqslant 0$. Then the set $S_{\mathbf{a}}$ of nonnegative integers $n$ such that $a_{n}=0$ is a union of at most finitely many arithmetic progressions along with a set of Banach density equal to 0 .

Proof. We define an $r$-by- $r$ matrix whose entries are rational functions:

$$
A(z):=\left(\begin{array}{cccccc}
0 & 1 & 0 & 0 & \ldots & 0 \\
0 & 0 & 1 & 0 & \ldots & 0 \\
\ldots & \ldots & \ldots & \ldots & \ldots & \ldots \\
-\frac{P_{0}(z)}{P_{r}(z)} & -\frac{P_{1}(z)}{P_{r}(z)} & \cdots & \ldots & \cdots & -\frac{P_{r-1}(z)}{P_{r}(z)}
\end{array}\right)
$$

Then the rational map $\Phi: \mathbb{A}^{1} \times \mathbb{A}^{r} \rightarrow \mathbb{A}^{1} \times \mathbb{A}^{r}$ (defined over $K$ ) by

$$
\Phi(z, v)=(z+1, A(z) \cdot v)
$$

has the property that for $v_{0} \in \mathbb{A}^{r}(K)$ given by $v_{0}:=\left(a_{0}, a_{1}, \cdots, a_{r-1}\right)$, we have $\Phi^{n}\left(0, v_{0}\right)=\left(n, a_{n}, a_{n+1}, \cdots, a_{n+r-1}\right)$.

Let $Y_{1}:=\{0\} \times \mathbb{A}^{r-1}$ and let $Y:=\mathbb{A} \times Y_{1}$. Applying Corollary 1.5 to the variety $X:=\mathbb{A}^{1} \times \mathbb{A}^{r}$, equipped with the rational self-map $\Phi$, to the subvariety $Y \subset X$ and to the point $\alpha:=\left(0, v_{0}\right) \in X(K)$, we obtain the desired conclusion.

If $K$ has characteristic 0 , then the same proof as above works under the weaker hypothesis that $P_{r}$ is not identically equal to 0 . Indeed, at the expense of considering the sequence of $a_{n}$ 's only for $n$ sufficiently large, we may assume $P_{r}(n) \neq 0$ as in Theorem 1.7. If $K$ has positive characteristic, one can easily see that the above result fails without the hypothesis that $P_{r}(n) \neq 0$ for all $n$.

If each polynomial $P_{j}$ is constant, then $\left\{a_{n}\right\}_{n \geqslant 0}$ is a linear recurrence sequence, and when $K$ has characteristic 0, the classical Skolem-Mahler-Lech theorem yields that the above set $S_{\mathbf{a}}$ is a union of at most finitely many arithmetic progressions along with a finite set. If $K$ has characteristic $p$, then there are examples (similar to the examples appearing in the Mordell-Lang problem in characteristic $p$ ) when $S_{\mathbf{a}}$ is an infinite set of Banach density 0. Now, in general, if $F(z):=\sum_{n=0}^{\infty} a_{n} z^{n}$ satisfies a differential equation with polynomial coefficients, then the sequence $\left\{a_{n}\right\}_{n \geqslant 0}$ satisfies a recurrence relation such as the one in Theorem 1.7. Rubel [21, Problem 16] asked whether the Skolem-Mahler-Lech theorem could be extended to such sequences $\left\{a_{n}\right\}_{n \geqslant 0}$, i.e. show that the set of all $n$ 's such that $a_{n}=0$ is a union of finitely many arithmetic progressions along with a finite set. First Bézivin [6] (under a technical assumption) and then Methfessel [19] (in general) answered Rubel's question in the affirmative except possibly for a set of natural density 0 (see Theorem 1.7). Both Bézivin and Methfessel used in their proofs the famous theorem of Szemerédi [22] regarding the existence of arbitrarily long arithmetic progressions in sets of positive natural density. We note that in order to prove our Theorem 1.4 we use Lemma 2.1 which is only reminiscent of Szemerédi's 
famous result, but which is technically different. We believe our Lemma 2.1 would be useful for other applications as well. Another advantage of our approach is that it provides slightly stronger results by replacing sets of natural density 0 with sets of Banach density 0.

Using a similar approach to that of Theorem 1.4 we are able to prove a result similar to Theorem 1.4 for the backward orbit of a point in a Noetherian space. More precisely, for a Noetherian space $X$, a continuous function $f: X \longrightarrow X$, and a point $x \in X$, we define a coherent backward orbit of $x$ (with respect to $f$ ) be a sequence $\left\{x_{-n}\right\}_{n \geqslant 0}$ such that

$$
x_{0}=x \quad \text { and } \quad f\left(x_{-n-1}\right)=x_{-n} \quad \text { for each } n \geqslant 0 .
$$

We obtain the following result.

Theorem 1.8. Let $X$ be a Noetherian space, let $f: X \longrightarrow X$ be a continuous function, let $\left\{x_{-n}\right\}_{n \geqslant 0}$ be a coherent backward orbit of a point $x \in X$, and let $Y \subseteq X$ be a closed set. Then the set $S:=\left\{n \in \mathbb{N}: x_{-n} \in Y\right\}$ is a union of at most finitely many arithmetic progressions and a set of Banach density zero.

In particular, we ask the following question for algebraic dynamical systems.

Question 1.9. Let $X$ be a quasi-projective variety defined over $\mathbb{C}$, let $\Phi: X \longrightarrow$ $X$ be an endomorphism, let $\left\{x_{-n}\right\}_{n \geqslant 0}$ be a coherent backward orbit of a point $x \in X(\mathbb{C})$ (with respect to $\Phi$ ), and let $Y \subseteq X$ be a subvariety. Is the set $S:=$ $\left\{n \in \mathbb{N}: x_{-n} \in Y(\mathbb{C})\right\}$ a union of at most finitely many arithmetic progressions?

Question 1.9 is related to the Dynamical Manin-Mumford Conjecture (see $[24,12])$. A positive answer to Question 1.9 yields that if a subvariety $Y$ contains a Zariski dense set of points in common with a coherent backward orbit of a point $x \in X(\mathbb{C})$, then $Y$ is periodic under the action of $\Phi$. In the original formulation (see [24]) of the Dynamical Manin-Mumford Conjecture, it was asked whether a subvariety $Y$ of a projective variety $X$ would have to be preperiodic under the action of a polarizable endomorphism $\Phi$ of $X$ if $Y$ contains a Zariski dense set of preperiodic points (we call a point $y \in X$ preperiodic if its orbit $\mathcal{O}_{\Phi}(x)$ is finite). If the point $x$ in Question 1.9 is preperiodic, then each point in a coherent backward orbit of $x$ is preperiodic, and thus a positive answer to Question 1.9 provides a positive answer to this special case of the Dynamical Manin-Mumford Conjecture. We note that there are counterexamples coming from endomorphisms of CM abelian varieties to the original formulation of the Dynamical Manin-Mumford Conjecture (see [12]), but we do not know whether those types of counterexamples can be found to Question 1.9.

While writing this paper we learned that, using different techniques, Clayton Petsche proved Theorem 1.4 when $\Phi$ is an endomorphism of an affine variety $X$. Petsche uses methods from topological dynamics and ergodic theory; in particular, he uses Berkovich spaces and a strong topological version of Furstenberg's Poincaré Recurrence Theorem. William Gignac indicated to us that Theorem 1.4 can also be derived using arguments that come from a deep result of ergodic theory on 
Noetherian spaces proved by Charles Favre (this is Théorème 2.5.8 in Favre's PhD thesis [10]); see also [15, Example A.3.2] and [16, Theorem 1.6] for an alternative proof of Favre's result using measure-theoretic methods. Gignac also told us that one can prove Theorem 1.8 by employing measure-theoretic techniques similar to those of $[15,16]$. We thank both Clayton Petsche and William Gignac for useful conversations on this topic. An advantage of our more elementary method for proving Theorem 1.4 (and the related results such as Theorem 1.8) is that it allows us to derive explicit bounds on the ratios of the infinite arithmetic progressions in some cases (see Theorems 4.1 and 4.2). Moreover, our approach allows us to derive a more precise result than Theorem 1.4 for the case of non-periodic curves.

Theorem 1.10. Let $C \subseteq \mathbb{P}^{\ell}$ be an irreducible curve of degree $d$ defined over a field $K$, let $\Phi: \mathbb{P}^{\ell} \longrightarrow \mathbb{P}^{\bar{\ell}}$ be an endomorphism of degree $m$ defined over $K$, and let $x \in \mathbb{P}^{\ell}(K)$ be a non-preperiodic point. If $C$ is not periodic, then there exists a constant $c_{0}$ depending only on $d, m$, and $\ell$ such that for all integers $N \geqslant 2$, we have

$$
\#\left\{1 \leqslant n \leqslant N: \Phi^{n}(x) \in C(K)\right\} \leqslant \frac{c_{0} N}{\log (N)} .
$$

We now briefly sketch the plan of this paper. In Section 2, we prove the key technical Lemma 2.1. In Section 3 we prove Proposition 3.1 (which is essentially Proposition 1.6 for Noetherian spaces) and then deduce various consequences such as Theorems 1.4 and 1.8. We conclude in Section 4 with some remarks on quantitative results, including the proofs of Theorems 4.1, 4.2 and 1.10.

\section{A technical lemma}

The key result for the proof of Proposition 1.6 and its related consequences is the following Lemma.

Lemma 2.1. Let $S$ be a set of positive integers having positive Banach density. Let $N \geqslant[1 / \delta(S)]+1$ be an integer, where $[x]$ as usual denotes the greatest integer less than or equal to $x$. Then there is a positive integer $k$ and a subset $Q \subseteq S$ such that

1. $k \leqslant N-1$;

2. $\delta(Q) \geqslant \frac{N \delta(S)-1}{2 N^{2}(N-1)}>0$; and

3. for all $a \in Q$, we have $a+k \in S$.

Proof. By assumption, $\frac{1}{N}<\delta(S)$. So there exist intervals $I_{n}$ with $\left|I_{n}\right| \rightarrow \infty$ such that

$$
\frac{\left|S \cap I_{n}\right|}{\left|I_{n}\right|}>\frac{\delta(S)+\frac{1}{N}}{2} .
$$

Let $P=\{i:|\{i N+1, \ldots,(i+1) N\} \cap S| \geqslant 2\}$. We claim that $P$ has positive Banach density. To see this, let $J_{n}=\left\{i:\{i N+1, \ldots,(i+1) N\} \subseteq I_{n}\right\}$. Then $\left|J_{n}\right| \leqslant$ $\frac{\left|I_{n}\right|}{N}$ and $\left|J_{n}\right| \rightarrow \infty$ as $n \rightarrow \infty$. For $i \in J_{n} \backslash P$ we have $S \cap\{i N+1, \ldots,(i+1) N\}$ has size at most 1 and for $i \in P \cap J_{n}$ we have $S \cap\{i N+1, \ldots,(i+1) N\}$ has size 
at most $N$. Since there are at most $2 N$ elements of $I_{n}$ that are not accounted for by taking the union of the $\{i N+1, \ldots,(i+1) N\}$ with $i \in J_{n}$, we see that

$$
\frac{\left(\delta(S)+\frac{1}{N}\right) \cdot\left|I_{n}\right|}{2} \leqslant\left|I_{n} \cap S\right| \leqslant\left|J_{n} \backslash P\right|+N\left|P \cap J_{n}\right|+2 N .
$$

Using the fact that $\left|J_{n}\right| \leqslant \frac{\left|I_{n}\right|}{N}$, we see

$$
\frac{\left(\delta(S)+\frac{1}{N}\right) \cdot N\left|J_{n}\right|}{2} \leqslant\left|J_{n} \backslash P\right|+N\left|P \cap J_{n}\right|+2 N .
$$

Dividing by $N\left|J_{n}\right|$ now gives

$$
\frac{\delta(S)+\frac{1}{N}}{2} \leqslant \frac{\left|J_{n} \backslash P\right|}{N\left|J_{n}\right|}+\frac{\left|P \cap J_{n}\right|}{\left|J_{n}\right|}+\frac{2}{\left|J_{n}\right|} .
$$

Since $\left|J_{n} \backslash P\right| \leqslant\left|J_{n}\right|$, we get

$$
\frac{\delta(S)+\frac{1}{N}}{2} \leqslant \frac{1}{N}+\frac{\left|P \cap J_{n}\right|}{\left|J_{n}\right|}+\frac{2}{\left|J_{n}\right|},
$$

which gives

$$
\frac{\delta(S)-\frac{1}{N}}{2} \leqslant \frac{\left|P \cap J_{n}\right|}{\left|J_{n}\right|}+\frac{2}{\left|J_{n}\right|} .
$$

Since $\left|J_{n}\right| \rightarrow \infty$, we see that $\delta(P) \geqslant \frac{\delta(S)-\frac{1}{N}}{2}$.

For each $i \in P$, we pick $a_{i}, b_{i} \in\{i N+1, \ldots,(i+1) N\} \cap S$ with $0<b_{i}-a_{i}<N$. For $j \in\{1, \ldots, N-1\}$, we let $P_{j}:=\left\{i \in P: b_{i}-a_{i}=j\right\}$. Then $P=\cup_{j=1}^{N-1} P_{j}$ and since Banach density is subadditive, we have

$$
\delta(P) \leqslant \sum_{j=1}^{N-1} \delta\left(P_{j}\right)
$$

Thus there is some $k \in\{1, \ldots, N-1\}$ such that $\delta\left(P_{k}\right) \geqslant \frac{\delta(P)}{N-1}$. Let $Q:=\left\{a_{i}: i \in\right.$ $\left.P_{k}\right\} \subseteq S$. Then $a+k \in S$ for all $a \in Q$ and a simple computation yields

$$
\delta(Q) \geqslant \frac{\delta\left(P_{k}\right)}{N} \geqslant \frac{N \delta(S)-1}{2 N^{2}(N-1)}>0
$$

as desired.

We find useful (see Theorem 4.2) stating the following Corollary of Lemma 2.1.

Corollary 2.2. Let $S$ be a set of positive integers having positive Banach density. Then there is a positive integer $k<\frac{2}{\delta(S)}$ and a subset $Q \subseteq S$ such that

(a) $\delta(Q) \geqslant \frac{\delta(S)^{3}}{24}$; and

(b) for all $a \in Q$, we have $a+k \in S$. 
Proof. We let $\delta:=\delta(S)$, and we apply Lemma 2.1 with $N=\left[\frac{2}{\delta}\right]$ (which is at least equal to $\left[\frac{1}{\delta}\right]+1$ since $\delta \leqslant 1$ ). This shows the existence of a set $Q \subseteq S$ satisfying property (b) above; in addition $\delta(Q) \geqslant \frac{N \delta-1}{2 N^{2}(N-1)}$. So, in order to show that (a) holds, it suffices to prove that $\frac{N \delta-1}{2 N^{2}(N-1)} \geqslant \frac{\delta^{3}}{24}$, which is equivalent with proving that

$$
\frac{N \delta-1}{N-1} \geqslant \frac{N^{2} \delta^{3}}{12}=\frac{2}{3 N} \cdot\left(\frac{N \delta}{2}\right)^{3} .
$$

Since $N=\left[\frac{2}{\delta}\right] \leqslant \frac{2}{\delta}$, then it suffices to show that $\frac{N \delta-1}{N-1} \geqslant \frac{2}{3 N}$, which is equivalent with showing that

$$
\delta \geqslant \frac{5}{3 N}-\frac{2}{3 N^{2}} .
$$

Because $N=\left[\frac{2}{\delta}\right]$, then $\frac{2}{\delta}<N+1$ and so, $\delta>\frac{2}{N+1}$. Then inequality (2.1) follows since

$$
\frac{2}{N+1}-\left(\frac{5}{3 N}-\frac{2}{3 N^{2}}\right)=\frac{N-5}{3 N(N+1)}+\frac{2}{3 N^{2}} \geqslant 0 .
$$

Inequality 2.2 is obvious for all $N \geqslant 5$, while for $N \in\{2,3,4\}$ the inequality can be checked directly (note that $N=\left[\frac{2}{\delta}\right] \geqslant 2$ because $\delta \leqslant 1$ ).

\section{Proof of our main results}

Theorem 1.4 will follow as a consequence of the following Proposition which is a generalization of Proposition 1.6.

Proposition 3.1. Let $X$ be a Noetherian topological space, let $\Phi: X \longrightarrow X$ be a continuous map, let $x \in X$, let $Y$ be a closed subset of $X$, and let $S:=$ $\left\{n: \Phi^{n}(x) \in Y\right\}$. If $S$ has positive Banach density, then it contains an infinite arithmetic progression.

Proof. Consider the set $\mathcal{V}$ of all closed subsets $V$ of $X$ with the property that $T_{V}:=\left\{n: \Phi^{n}(x) \in V\right\}$ has positive Banach density but does not contain an infinite arithmetic progression. If $\mathcal{V}$ is empty, then there is nothing to prove. Thus we may assume, towards a contradiction, that $\mathcal{V}$ is non-empty. We let $W$ be a minimal element of $\mathcal{V}$ with respect to the inclusion of sets (note that such an element exists since $X$ is Noetherian). By Lemma 2.1, we have a positive integer $k$ and a subset $Q \subseteq T_{W}$ with $\delta(Q)>0$ such that $a+k \in T_{W}$ for all $a \in Q$.

If $n \in Q$, then $\Phi^{n}(x) \in W$ and $\Phi^{n+k}(x) \in W$. Thus $\Phi^{n}(x) \in W \cap \Phi^{-k}(W)$ whenever $n \in Q$. If $\Phi^{-k}(W) \supseteq W$ then $T_{W}$ has the property that $n+k \in T_{W}$ whenever $n \in T_{W}$ and since $T_{W}$ is non-empty, it contains an infinite arithmetic progression, which contradicts the fact that $W \in \mathcal{V}$. Thus $Z:=W \cap \Phi^{-k}(W)$ is a proper closed subset of $W$ (since $\Phi$ is continuous and $W$ is closed) and so we have $\Phi^{n}(x) \in Z$ for all $n \in Q$. Since $Q$ has positive Banach density, we obtain that $T_{Z} \supseteq Q$ also has positive Banach density and therefore $T_{Z}$ contains an infinite arithmetic progression. Since $T_{Z} \subseteq T_{W}$, we see that $T_{W}$ contains an infinite arithmetic progression, a contradiction. This concludes our proof. 
Theorem 1.4 follows easily now.

Proof of Theorem 1.4. Suppose not. Let $\mathcal{V}$ be the collection of all closed subsets $V$ of $X$ that have the property that there is some continuous map $g: V \rightarrow V$, and some closed subset $W$ of $V$, and a point $y \in V$ such that $\left\{n: g^{n}(y) \in W\right\}$ cannot be expressed as a finite union of arithmetic progressions along with a set of Banach density zero.

By assumption, $X \in \mathcal{V}$ and so we may choose a minimal element $V \in \mathcal{V}$. Then there is some continuous map $g: V \longrightarrow V$, some closed subset $W$ of $V$, and some point $y \in V$ such that $T:=\left\{n: g^{n}(y) \in W\right\}$ cannot be expressed as a finite union of arithmetic progressions along with a set of Banach density zero. We necessarily have that $W_{i}:=g^{-i}(W)$ is a proper closed subset of $V$ (note that $g$ is continuous), since otherwise $T$ would contain every integer greater than or equal to $i$ (and thus it would be the union of an arithmetic progression with a finite set). Moreover, by our choice of $V, W$ and $y$, it follows that $\delta(T)>0$ and thus by Proposition 3.1, there exist $a, b \in \mathbb{N}$ such that $T \supseteq\{a n+b: n \geqslant 0\}$. Let $C_{i}$ denote the closure of $S_{i}:=\left\{g^{(a n+b)}(y): n \geqslant i\right\}$. Then

$$
C_{0} \supseteq C_{1} \supseteq \cdots
$$

is a descending chain of closed sets and hence there is some $m$ such that $C_{m}=$ $C_{m+1}=\cdots$. We take $V_{0}=C_{m}$. Then $g^{-a}\left(V_{0}\right) \supseteq g^{-a}\left(S_{m+1}\right) \supseteq S_{m}$ and since $g^{-a}\left(V_{0}\right)$ is closed we thus see it contains the closure of $S_{m}$, which is $V_{0}$.

Then $V_{0} \subseteq W$ is closed and we have $g^{-a}\left(V_{0}\right) \supseteq V_{0}$. We let $V_{j}$ denote the closed set $g^{-j}\left(V_{0}\right)$ for $j \in\{1, \ldots, a-1\}$. Since $V_{j} \subseteq W_{a+j} \subsetneq V$, we see that each $V_{j}$ is a proper subset for $0 \leqslant j \leqslant a-1$. Then $g^{-a}\left(V_{j}\right)=g^{-a}\left(g^{-j}\left(V_{0}\right)\right)=g^{-j}\left(g^{-a}\left(V_{0}\right)\right) \supseteq$ $g^{-j}\left(V_{0}\right)=V_{j}$, and so for $j \in\{0, \ldots, a-1\}$, we have $g^{-j+n a+b}(y) \in V_{j}$ for every $n>m$. Moreover, since $g^{-a}\left(V_{j}\right) \supseteq V_{j}$, we have that $h:=g^{a}$ restricts to continuous maps $h: V_{j} \longrightarrow V_{j}$ for each $0 \leqslant j \leqslant a-1$. We let $y_{j}:=g^{-j+a+b}(y)$. It follows from the minimality of $V$ that

$$
T_{j}:=\left\{n \geqslant m: h^{n}\left(y_{j}\right) \in W \cap V_{j}\right\}
$$

is a finite union of arithmetic progressions along with a set of Banach density zero. On the other hand,

$$
T_{j}=\left\{n \geqslant m: g^{-j+a(n+1)+b}(y) \in W\right\},
$$

for each $j=0, \cdots, a-1$. Then, up to a finite set, we have

$$
T=\bigcup_{j=0}^{a-1}\left(a T_{j}+b+a-j\right),
$$

where for any set $U \subseteq \mathbb{N}$ and any $c \in \mathbb{N}$, we let $c \cdot U$ be the set $\{c j: j \in U\}$, and we let $c+U:=U+c$ be the set $\{c+j: j \in U\}$. Hence $T$ is a finite union of arithmetic progressions along with a set of Banach density zero. 
As a consequence to our main result, we can prove the following (seemingly) stronger statement.

Theorem 3.2. Let $X$ be a Noetherian space, let $U \subseteq X$ be an open subset, and let $\Phi: U \longrightarrow X$ be a continuous map. Let $x \in X$ such that $\Phi^{n}(x) \in U$ for each nonnegative integer $n$. Then for each closed set $Y \subseteq X$, the set $S:=\{n \in$ $\left.\mathbb{N}: \Phi^{n}(x) \in Y\right\}$ is a union of at most finitely many arithmetic progressions along with a set of Banach density zero

Proof. Let $Z:=\cap_{n \geqslant 0} \Phi^{-n}(U)$; we know that $Z$ is nonempty since $x$ (and therefore $\left.\mathcal{O}_{\Phi}(x)\right)$ is contained in $Z$. We endow $Z$ with the inherited topology from $X$; then $Z$ is also a Noetherian space. Furthermore, by its definition, we obtain that $\Phi$ restricts to a self-map $\Phi_{Z}: Z \longrightarrow Z$. Next we show that $\Phi_{Z}$ is continuous.

Indeed, let $V \subseteq X$ be an open set and we need to show that $\Phi_{Z}^{-1}(V \cap Z)$ is open in $Z$. This follows immediately once we prove that $\Phi_{Z}^{-1}(V \cap Z)=\Phi^{-1}(V) \cap Z$ because $\Phi: U \longrightarrow X$ is continuous and so $\Phi^{-1}(V)$ is open in $U$ and (because $U$ is an open subset of $X)$ it is also open in $X$ which yields that $\Phi^{-1}(V) \cap Z$ is open in $Z$. To see that $\Phi_{Z}^{-1}(V \cap Z)=\Phi^{-1}(V) \cap Z$ we note that for each $y \in \Phi_{Z}^{-1}(V \cap Z) \subseteq Z$ we have $\Phi_{Z}(y) \in V$. So, $\Phi(y) \in V$ and thus $y \in \Phi^{-1}(V) \cap Z$. Conversely, if $y \in \Phi^{-1}(V) \cap Z$, then $y \in Z$ and so $\Phi_{Z}(y) \in V \cap Z$ as claimed.

Therefore $\Phi_{Z}: Z \longrightarrow Z$ is a continuous map on a Noetherian space. Hence by Theorem 1.4, the set of all $n \in \mathbb{N}$ such that $\Phi_{Z}^{n}(x) \in Y \cap Z$ is a union of at most finitely many arithmetic progressions along with a set of Banach density zero. Because $\Phi=\Phi_{Z}$ on $Z$ then $\Phi^{n}(x) \in Y$ if and only if $\Phi_{Z}^{n}(x) \in Y \cap Z$, which concludes our proof.

Corollary 1.5 is an immediate consequence of Theorem 3.2. The proof for Theorem 1.8 is similar to the proof of Theorem 1.4 and it relies on the following result.

Proposition 3.3. Let $X$ be a Noetherian space, let $f: X \longrightarrow X$ be a continuous function, let $\left\{x_{-n}\right\}_{n \geqslant 0}$ be a coherent backward orbit of a point $x \in X$, and let $Y \subseteq X$ be a closed set. If the set $S:=\left\{n \in \mathbb{N}: x_{-n} \in Y\right\}$ has positive Banach density then it contains an infinite arithmetic progression.

Proof. The proof is similar to the proof of Proposition 3.1. Consider the set $\mathcal{V}$ of all closed subsets $V$ of $X$ with the property that $T_{V}:=\left\{n: x_{-n} \in V\right\}$ has positive Banach density but does not contain an infinite arithmetic progression. If $\mathcal{V}$ is empty, then there is nothing to prove. Thus we may assume, towards a contradiction, that $\mathcal{V}$ is non-empty. We let $W$ be a minimal element of $\mathcal{V}$ with respect to the inclusion of sets (note that such an element exists since $X$ is Noetherian). By Lemma 2.1, we see that there exists a positive integer $k$ and a set $Q \subseteq T_{W}$ of positive Banach density such that if $n \in Q$ then $n+k \in T_{W}$. Thus $x_{-n-k} \in W \cap f^{-k}(W)$ whenever $n \in Q$. There are two cases: either $f^{-k}(W) \supseteq W$ or not.

Assume now that $f^{-k}(W) \supseteq W$; so if $y \in W$, then also $f^{k}(y) \in W$. Then $T_{W}$ has the property that $n-k \in T_{W}$ whenever $n \in T_{W}$ and $n \geqslant k$. Because 
$Q$ has positive Banach density, then it is infinite, and therefore there exists $j \in$ $\{0, \ldots, k-1\}$ such that there exist infinitely many $n \in Q$ satisfying $n \equiv j(\bmod k)$. So there exists a sequence of integers $n_{i} \rightarrow \infty$ contained in $T_{W}$ such that $n_{i} \equiv j$ $(\bmod k)$ for each $i$ and moreover, $n_{i}-\ell k \in T_{W}$ for all $\ell \geqslant 0$ such that $n_{i}-\ell k \geqslant 0$. Thus $T_{W}$ contains the infinite arithmetic progression $\{j+\ell k\} \ell \geqslant 0$, as desired.

Assume now that $W \nsubseteq f^{-k}(W)$. Then $Z:=W \cap f^{-k}(W)$ is a proper closed subset of $W$ and so we have $x_{-n-k} \in Z$ for all $n \in Q$. Since $Q$ has positive Banach density, we obtain that also $T_{Z}$ has positive Banach density (note that $\delta(Q+k)=\delta(Q)>0$ and $\left.(Q+k) \subseteq T_{Z}\right)$. Hence $T_{Z}$ contains an infinite arithmetic progression (because $Z$ is a proper subset of $W$ ). Since $T_{Z} \subseteq T_{W}$, we see that $T_{W}$ contains an infinite arithmetic progression, a contradiction. This concludes our proof.

Theorem 1.8 follows from Proposition 3.3 similar to the proof of Theorem 1.4.

Proof of Theorem 1.8. Let $\mathcal{V}$ be the collection of all closed subsets $V$ of $X$ that have the property that there is some continuous map $g: V \rightarrow V$, and some closed subset $W$ of $V$, and a coherent backward orbit $\left\{y_{-n}\right\}$ of a point $y \in V$ such that $\left\{n: y_{-n} \in W\right\}$ cannot be expressed as a finite union of arithmetic progressions along with a set of Banach density zero.

Assume, for contradiction, that $X \in \mathcal{V}$ and so we may choose a minimal element $V \in \mathcal{V}$. Then there is some continuous map $g: V \longrightarrow V$, some closed subset $W$ of $V$, and a coherent backward orbit $\left\{y_{-n}\right\}_{n \geqslant 0}$ of a point $y \in V$ such that $T:=$ $\left\{n: y_{-n} \in W\right\}$ cannot be expressed as a finite union of arithmetic progressions along with a set of Banach density zero.

For each $i \in \mathbb{N}$ we let $W_{i}:=g^{-i}(W)$. We claim that if $W_{i}=V$ for some $i \in \mathbb{N}$, then $T$ is a union of at most finitely many arithmetic progressions along with a finite set (which obviously has Banach density zero). Indeed, for each $j \in\{0, \ldots, i-1\}$ we let $T_{i, j}:=\{n \in T: n \equiv j(\bmod i)\}$. Then $T=\cup_{j=0}^{i-1} T_{i, j}$. Assume $T_{i, j}$ is infinite (for some $0 \leqslant j \leqslant i-1$ ). Since $W_{i}=V$ then for each $n \in T$ also $n-i \in T$ and therefore $n-i \ell \in T$ for all $\ell \geqslant 0$ such that $n-i \ell \geqslant 0$. Because we assumed that $T_{i, j}$ is infinite, then $j+i \ell \in T$ for all $\ell \geqslant 0$. In conclusion, $T$ is indeed a union of at most finitely many arithmetic progressions (of ratio $i$ ) along with a finite set.

So from now on assume that $W_{i}:=g^{-i}(W)$ is a proper closed subset of $V$ (note that $g$ is continuous). Moreover, by our choice of $V, W$ and $y$, it follows that $\delta(T)>0$ and thus by Proposition 3.3, there exist $a, b \in \mathbb{N}$ such that $T \supseteq$ $\{a n+b: n \geqslant 0\}$. Let $C_{i}$ denote the closure of $S_{i}:=\left\{y_{-(a n+b)}: n \geqslant i\right\}$. Then

$$
C_{0} \supseteq C_{1} \supseteq \cdots
$$

is a descending chain of closed sets and hence there is some $m$ such that $C_{m}=$ $C_{m+1}=\cdots$. We take $V_{0}=C_{m}$. Then $g^{-a}\left(V_{0}\right) \supseteq g^{-a}\left(S_{m}\right) \supseteq S_{m+1}$ and since $g^{-a}\left(V_{0}\right)$ is closed we thus see it contains the closure of $S_{m+1}$, which is $V_{0}$.

Then $V_{0} \subseteq W$ is closed and we have $g^{-a}\left(V_{0}\right) \supseteq V_{0}$. We let $V_{j}$ denote the closed set $g^{-j}\left(V_{0}\right)$ for $j \in\{1, \ldots, a-1\}$. Since $V_{j} \subseteq W_{a+j} \subsetneq V$, we see that each $V_{j}$ is 
a proper subset for $0 \leqslant j \leqslant a-1$. Then $g^{-a}\left(V_{j}\right)=g^{-a}\left(g^{-j}\left(V_{0}\right)\right)=g^{-j}\left(g^{-a}\left(V_{0}\right)\right) \supseteq$ $g^{-j}\left(V_{0}\right)=V_{j}$, and so for $j \in\{0, \ldots, a-1\}$, we have $y_{-(n a+b+j)} \in V_{j}$ for every $n \geqslant m$. Moreover, since $g^{-a}\left(V_{j}\right) \supseteq V_{j}$, we have that $h:=g^{a}$ restricts to continuous maps $h: V_{j} \longrightarrow V_{j}$ for each $0 \leqslant j \leqslant a-1$. It follows from the minimality of $V$ that

$$
T_{j}:=\left\{n \geqslant m:\left(y_{-(n a+b+j)}\right) \in W \cap V_{j}\right\}
$$

is a finite union of arithmetic progressions along with a set of Banach density zero (since $\left\{y_{-j-n a-b}\right\}_{n \geqslant 0}$ is a coherent backward orbit of $y_{-j-b}$ under the action of $h)$. Then, up to a finite set, we have

$$
T=\bigcup_{j=0}^{a-1}\left(a T_{j}+b+j\right)
$$

and hence $T$ is a finite union of arithmetic progressions along with a set of Banach density zero.

\section{Some quantitative results}

The following result is an easy application of Lemma 2.1 .

Theorem 4.1. Let $X$ be a quasi-projective variety defined over a field $K$, let $\Phi: X \longrightarrow X$ be an endomorphism defined over $K$, let $C \subseteq X$ be an irreducible curve, and let $\alpha \in X(K)$ be a point that is not preperiodic under $\Phi$. If the set $S:=\left\{n \in \mathbb{N}: \Phi^{n}(\alpha) \in C(K)\right\}$ has Banach density $\delta>0$, then $S$ contains an infinite arithmetic progression of ratio $k=\frac{1}{\delta}$, and $\Phi^{k}(C) \subseteq C$.

Proof. It follows from Lemma 2.1 applied with $N=\left[\frac{1}{\delta}\right]+1$ that there exists a positive integer $k \leqslant\left[\frac{1}{\delta}\right]$ and a subset $Q \subset S$ of positive density such that for each $n \in Q$, also $\Phi^{n+k}(\alpha) \in C(K)$. So $\Phi^{n}(\alpha) \in C \cap \Phi^{-k}(C)$. Hence, $C \cap \Phi^{-k}(C)$ contains an infinite set of points (because $\alpha$ is not preperiodic under the action of $\Phi)$. Since $C$ is an irreducible curve, we see then that $C \subseteq \Phi^{-k}(C)$; thus $\Phi^{k}(C) \subseteq C$. This yields the desired infinite arithmetic progression of ratio $k \leqslant \frac{1}{\delta}$. If $k<1 / \delta$, then the existence of this arithmetic progression would imply that $\delta \geqslant 1 / k>\delta$, a contradiction. Thus, $k=\frac{1}{\delta}$.

Applying the technique of the proof of Theorem 4.1 recursively in the case of endomorphisms of $\mathbb{P}^{\ell}$ one can obtain a similar result for all projective subvarieties of $\mathbb{P}^{\ell}$. This may be viewed as a weak dynamical analog of a result of Evertse-Schlickewei-Schmidt [8, Theorem 1.2] on effective bounds for the ratios of arithmetic progressions that arise from linear recurrence sequences.

Theorem 4.2. For each $\delta>0, D \geqslant 1$ real numbers, and each $\ell, e, m \in \mathbb{N}$, there exists a positive real number $M:=M(\delta, m, D, \ell, e)$ with the following property: for any endomorphism $\Phi: \mathbb{P}^{\ell} \longrightarrow \mathbb{P}^{\ell}$ given by homogenous polynomials of degree at 
most $m$ defined over any algebraically closed field $K$, any irreducible subvariety $V \subseteq \mathbb{P}^{\ell}$ of dimension at most $e$ and degree at most $D$, and any $x \in \mathbb{P}^{\ell}(K)$, if the set $S:=\left\{n \in \mathbb{N}: \Phi^{n}(\alpha) \in V(K)\right\}$ has Banach density at least equal to $\delta$, then $S$ contains an infinite arithmetic progression of ratio at most $M$.

Proof. One uses induction on the dimension $e$ of $V$, with the base case being Theorem 4.1; so $M(\delta, m, D, \ell, 1)=\frac{1}{\delta}$. Assume now $e \geqslant 2$. If $V \subseteq \Phi^{-k}(V)$ for some integer $k<N:=\left[\frac{2}{\delta}\right]$, then $V$ is periodic under $\Phi$ and moreover it contains an infinite arithmetic progression of ratio at most $\left[\frac{2}{\delta}\right]$. So, assume $V \cap \Phi^{-k}(V)$ has dimension at most $e-1$ for each $k<N$. By Corollary 2.2 applied with $N=\left[\frac{2}{\delta}\right]$, there is a set $Q \subset S$ of density at least equal to $\frac{\delta^{3}}{24}$ and there is an integer $k<N$ such that $\Phi^{n}(x) \in V \cap \Phi^{-k}(V)$ for each $n \in Q$. Then with the use of generalized Bézout's Theorem (see [18, Theorem 1], for example), one sees that the degree of $V \cap \Phi^{-k}(V)$ is at most $m^{k(\ell-e)} D^{2}$ (since $\Phi^{-k}(V)$ has degree at most $\left.m^{k(\ell-e)} D\right)$. Hence, by the pigeonhole principle, there exists an irreducible subvariety $W \subseteq V \cap \Phi^{-k}(V)$ of dimension at most $e-1$ such that the set $S_{W}:=\left\{n \in \mathbb{N}: \Phi^{n}(x) \in W(K)\right\}$ has density at least equal to $\frac{\delta^{3}}{24 m^{k(\ell-e)} D^{2}}$. Then the induction hypothesis yields the following recursive formula

$$
M(\delta, m, D, \ell, e)=M\left(\frac{\delta^{3}}{24 m^{\frac{2(\ell-e)}{\delta}} D^{2}}, m, m^{\frac{2(\ell-e)}{\delta}} D^{2}, \ell, e-1\right) .
$$

We conclude by proving Theorem 1.10.

Proof of Theorem 1.10. The proof relies on the following quantitative version of the argument from the proof of Theorem 4.1.

Lemma 4.3. Let $k \in \mathbb{N}$, and let $K, C, \Phi, x, m, d$ be as in Theorem 1.10. Let $c_{1}=2 m^{\ell} d^{2}$. Then we have

$$
\#\left\{n \geqslant 1: \Phi^{n}(x) \in C(K) \text { and } \Phi^{n+k}(x) \in C(K)\right\} \leqslant c_{1}^{k} .
$$

Proof of Lemma 4.3. Let $n$ be a positive integer such that $\Phi^{n}(x) \in C(K)$ and also $\Phi^{n+k}(x) \in C(K)$; then $\Phi^{n}(x) \in C \cap \Phi^{-k}(C)$. We know that $C$ is an irreducible non-periodic curve; then $C \cap \Phi^{-k}(C)$ is a proper intersection of projective curves, and therefore, by Bézout's Theorem (see [18, Theorem 1], for example), the number of points in the intersection is bounded above by

$$
\operatorname{deg}(C) \cdot \operatorname{deg}\left(\Phi^{-k}(C)\right) \leqslant\left(2 m^{\ell} d^{2}\right)^{k}=c_{1}^{k} .
$$

Based on Lemma 4.3, the rest of the proof is a simple counting argument. We let $\left\{n_{k}\right\}_{k \geqslant 1}$ be the increasing sequence of positive integers $n$ for which $\Phi^{n}(x) \in$ $C(K)$. The proof of Theorem 1.10 is a simple consequence of the following lemma.

Lemma 4.4. With notation as in Lemma 4.3, let $c_{2}=\left(4 c_{1} \log \left(c_{1}\right) \log _{c_{1}}\left(2 c_{1}\right)\right)^{-1}$. Then $n_{k} \geqslant c_{2} k \log (k)$ for each $k \geqslant 1$. 
Proof of Lemma 4.4. We first estimate the size of $n_{k}$ for $k>\left(2 c_{1}\right)^{4 c_{1}}$. Note that $c_{1} \geqslant 2$. Then there exists a unique positive integer $j$ such that

$$
\sum_{i=1}^{j} c_{1}^{i}<k \leqslant \sum_{i=1}^{j+1} c_{1}^{i}
$$

which, since $c_{1} \geqslant 2$, gives

$$
k \leqslant 2 c_{1}^{j+1}, \quad \text { or equivalently } \quad c_{1}^{j} \geqslant k / 2 c_{1} .
$$

Using Lemma 4.3, we obtain

$$
n_{k}=n_{1}+\sum_{i=1}^{k-1}\left(n_{i+1}-n_{i}\right) \geqslant \sum_{i=1}^{j} i \cdot c_{1}^{i} \geqslant j c_{1}^{j} .
$$

Then, using Equation (4.1), we find

$$
\begin{aligned}
n_{k} & \geqslant j c_{1}^{j} \geqslant \frac{k}{2 c_{1}} \log _{c_{1}}\left(k / 2 c_{1}\right)=k\left(\log _{c_{1}} k\right) \frac{\left(1-\log _{c_{1}}\left(2 c_{1}\right) / \log _{c_{1}}(k)\right)}{2 c_{1}} \\
& \geqslant \frac{1}{4 c_{1}} k \log _{c_{1}}(k),
\end{aligned}
$$

where the last inequality follows from the fact that we are assuming for now that $k>\left(2 c_{1}\right)^{4 c_{1}}$.

Suppose now that $k \leqslant\left(2 c_{1}\right)^{4 c_{1}}$. Since $n_{i} \geqslant i$ for every $i$, we see then that

$$
n_{k} \geqslant k \geqslant \frac{1}{4 c_{1} \log _{c_{1}}\left(2 c_{1}\right)} k \log _{c_{1}}(k) .
$$

Hence we have $n_{k} \geqslant c_{2} k \log (k)$ for every $k \geqslant 1$.

Now, for each $N \geqslant 2$, if $k:=\#\left\{1 \leqslant n \leqslant N: \Phi^{n}(x) \in C(K)\right\}$ then $n_{k} \leqslant N$. Using Lemma 4.4 we obtain

$$
N \geqslant n_{k} \geqslant c_{2} k \log (k)
$$

and therefore, $k \leqslant \frac{c_{0} N}{\log (N)}$ with $c_{0}:=\frac{2}{c_{2}}$ (which depends on $d, m$ and $\ell$ only).

\section{References}

[1] J.P. Bell, A generalized Skolem-Mahler-Lech theorem for affine varieties, J. London Math. Soc. (2) 73 (2006), 367-379.

[2] J.P. Bell, D. Ghioca, and T.J. Tucker, The dynamical Mordell-Lang problem for étale maps, Amer. J. Math. 132 (2010), 1655-1675.

[3] R.L. Benedetto, D. Ghioca, P. Kurlberg, and T.J. Tucker, A gap principle for dynamics, Compositio Math. 146 (2010), 1056-1072. 
[4] R.L. Benedetto, D. Ghioca, P. Kurlberg, and T.J. Tucker, A case of the dynamical Mordell-Lang conjecture (with an Appendix by U. Zannier), Math. Ann. 352 (2012), 1-26.

[5] R.L. Benedetto, D. Ghioca, B.A. Hutz, P. Kurlberg, T. Scanlon, and T.J. Tucker, Periods of rational maps modulo primes, Math. Ann. 355 (2013), 637-660.

[6] J.-P. Bézivin, Une généralisation du théorème de Skolem-Mahler-Lech, Quart. J. Math. Oxford Ser. (2) 40 (1989), 133-138.

[7] L. Denis, Géométrie et suites récurrentes, Bull. Soc. Math. France 122 (1994), $13-27$.

[8] J.-H. Evertse, H.P. Schlickewei, and W.M. Schmidt, Linear equations in variables which lie in a multiplicative group, Ann. of Math. (2) 155 (2002), 807836.

[9] N. Fakhruddin, The algebraic dynamics of generic endomorphisms of $\mathbb{P}^{n}$, to appear in Algebra \& Number Theory, 2013.

[10] C. Favre, Dynamique des applications rationnelles, $\mathrm{PhD}$ thesis, Université Paris-Sud XI - Orsay, 2000.

[11] D. Ghioca and T.J. Tucker, Periodic points, linearizing maps, and the dynamical Mordell-Lang problem, J. Number Theory 129 (2009), 1392-1403.

[12] D. Ghioca, T.J. Tucker and S. Zhang, Towards a dynamical Manin-Mumford Conjecture, IMRN 22 (2011), 5109-5122.

[13] D. Ghioca, T.J. Tucker, and M.E. Zieve, Intersections of polynomial orbits, and a dynamical Mordell-Lang conjecture, Invent. Math. 171 (2008), 463-483.

[14] D. Ghioca, T.J. Tucker, and M.E. Zieve, Linear relations between polynomial orbits, Duke Math. J. 161 (2012), 1379-1410.

[15] W. Gignac, Equidistribution of preimages in nonarchimedean dynamics, $\mathrm{PhD}$ thesis, University of Michigan, 2013.

[16] W. Gignac, Measures and dynamics on Noetherian spaces, to appear in J. Geom. Anal.

[17] R. Hartshorne, Algebraic geometry, Graduate Texts in Mathematics 52, Springer-Verlag, New York-Heidelberg, 1977. xvi+496 pp.

[18] J. Heintz, Definability and fast quantifier elimination in algebraically closed fields, Theoret. Comput. Sci. 24 (1983), no. 3, 239-277.

[19] C. Methfessel, On the zeros of recurrence sequences with non-constant coefficients, Arch. Math. (Basel) $\mathbf{7 4}$ (2000), 201-206.

[20] C. Petsche, On the distribution of orbits in affine varieties, preprint, arXiv:11401.3425.

[21] L.A. Rubel, Some research problems about algebraic differential equations, Trans. Amer. Math. Soc. 280 (1983), 43-52.

[22] E. Szemerédi, On sets of integeres containing no $k$ elements in arithmetic progression, Acta Arith. 27 (1975), 199-245.

[23] J. Xie, Dynamical Mordell-Lang Conjecture for birational polynomial morphisms on $\mathbb{A}^{2}$, to appear in Math. Ann.

[24] S. Zhang, Distributions in algebraic dynamics, Survey in Differential Geometry, vol. 10, International Press, 2006, pp. 381-430. 
Addresses: Jason P. Bell: Department of Pure Mathematics, University of Waterloo, Waterloo, ON N2L 3G1, Canada;

Dragos Ghioca: Department of Mathematics, University of British Columbia, Vancouver, BC V6T 1Z2, Canada;

Thomas J. Tucker: Department of Mathematics, University of Rochester, Rochester, NY 14627, USA.

E-mail: jpbell@uwaterloo.ca,dghioca@math.ubc.ca, ttucker@math.rochester.edu

Received: 24 November 2014; revised: 28 May 2015 